\title{
Some results on the stability of positive switched linear systems
}

\author{
Oliver Mason and Robert Shorten
}

\begin{abstract}
In this paper, we present a number of results concerned with the stability of positive switched linear systems. In particular, we show that a recent conjecture concerning the existence of common quadratic Lyapunov functions (CQLFs) for positive LTI systems is true for second order systems, and establish a class of switched linear systems for which CQLF existence is equivalent to exponential stability under arbitrary switching. However, this conjecture is false for higher dimensional systems and we illustrate this fact with a counterexample. A number of stability criteria for positive switched linear systems based on common diagonal Lyapunov functions (CDLFs) are also presented, as well as a necessary and sufficient condition for a general pair of positive LTI systems to have a CDLF. To the best of the authors' knowledge, this is the first time that a necessary and sufficient condition for CDLF existence for $n$-dimensional systems has appeared in the literature.
\end{abstract}

\section{INTRODUCTION}

The theory of positive LTI systems has historically assumed a position of great importance in systems theory and has been applied in the study of a wide variety of dynamic systems [1], [2], [3], [4]. Recently, new application studies in congestion control of the Internet [5], formation flying [6], and other areas, have highlighted the importance of time-varying positive linear systems (PLS) and, in particular, positive switched linear systems. While positive LTI systems are well understood, the basic properties of positive switched linear systems have yet to be ascertained. The most important of these concerns their stability and in this paper we report the results of some initial work on the stability of positive switched linear systems.

Our main concern throughout the paper is the problem of determining conditions that guarantee the stability of positive switched linear systems under arbitrary switching. In this context, it is well-known that the existence of a common quadratic Lyapunov function (CQLF) for the LTI systems $\Sigma_{A_{i}}: \dot{x}=A_{i} x \quad 1 \leq i \leq k$, is sufficient for the exponential stability, under arbitrary switching, of the associated switched linear system

$$
\Sigma_{S}: \dot{x}=A(t) x \quad A(t) \in\left\{A_{1}, \ldots, A_{k}\right\} .
$$

Formally, $V(x)=x^{T} P x$ is a CQLF for $\Sigma_{A_{i}}, 1 \leq i \leq k$, if $P=P^{T}>0$ and $A_{i}^{T} P+P A_{i}<0$ for $1 \leq i \leq k$. While CQLF existence is not in general necessary for the exponential stability of switched linear systems [7], recent work has established a number of system classes

O. Mason is with the Hamilton Institute, NUI Maynooth, Co. Kildare, Ireland oliver.mason@may. ie

R. Shorten is with the Hamilton Institute, NUI Maynooth, Co. Kildare, Ireland robert. shorten@may. ie for which this is not necessarily the case [8], [9]. It is the authors' belief that the identification of such system classes is a highly interesting and important problem. Some initial results and extensive numerical simulations led to the conjecture, presented in [10], that a necessary and sufficient condition for a pair of stable positive LTI systems $\Sigma_{A_{1}}$, $\Sigma_{A_{2}}$, to have a CQLF was that the product $A_{1} A_{2}^{-1}$ has no negative real eigenvalues. This would mean that CQLF existence was not conservative for the exponential stability of positive switched linear systems. We show below that while it is indeed true for pairs of second order positive LTI systems, the general conjecture for higher order systems is false.

As well as CQLFs, two other types of Lyapunov functions arise naturally when considering positive linear systems; namely diagonal Lyapunov functions and copositive Lyapunov functions [11]. The function $V(x)=x^{T} D x$ is a common diagonal Lyapunov function (CDLF) for the positive LTI systems, $\Sigma_{A_{i}}, 1 \leq i \leq k$, if $D$ is diagonal, positive definite and satisfies

$$
A_{i}^{T} D+D A_{i}<0, \quad 1 \leq i \leq k .
$$

While for general LTI systems, the problem of determining whether or not a given system has a diagonal Lyapunov function is extremely difficult [12], [13], any stable positive LTI system is known to have such a Lyapunov function [3], [2]. This fact naturally gives rise to the possibility of basing stability criteria for positive switched linear systems on the existence of CDLFs, and to the question of whether or not it is possible to obtain necessary and sufficient conditions for a family of positive LTI systems to have a CDLF.

In this paper we present some simple sufficient conditions for the stability of positive switched linear systems based on CDLFs and also, in Theorem 5.2 below we derive, following similar arguments to those presented in [14], an algebraic condition that is necessary and sufficient for a pair of positive LTI systems to have a CDLF. While the obtained condition is not simple to check, it does provide insight into the existence of such functions and it is hoped that it will provide a basis for the development of useful stability criteria for positive switched linear systems.

Before proceeding we note that the existence of diagonal Lyapunov functions is an important research area in its own right, and that several papers have appeared on this topic [12], [13], [15], [16], [17]. These functions arise in the study of decentralised and interconnected systems, [18], [19], as well as in the study of neural networks, and asynchronous computation [20]. The existence question for 
common diagonal Lyapunov functions arises naturally as the study of such systems extends to interconnections of time-varying and switched systems.

Traditionally, the conditions for CQLF and CDLF existence are imposed globally in the state space. This is unduly restrictive for positive systems as the state is constrained to the non-negative orthant. Thus, it is enough to require the existence of a function $x^{T} P x$ such that $x^{T} P x$ is positive, and $x^{T}\left(A_{i}^{T} P+P A_{i}\right) x$ is negative for all non-zero $x$ in the non-negative orthant, for $1 \leq i \leq k$. Such a function is called a common quadratic copositive Lyapunov function. We shall also briefly consider the existence question for such functions here, giving necessary and sufficient conditions for existence in the case of pairs of second order systems.

The paper is organised as follows. In Section 2, we introduce the notation used through the paper as well as some preliminary results that are used later. Then in Section 3 we show that the conjecture of [10] is true for second order systems and provide a counterexample demonstrating that it is false for higher order systems. We then turn our attention to stability criteria based on common diagonal Lyapunov functions and after giving some simple sufficient conditions for CDLF existence in Section 4, we derive, for the first time, a necessary and sufficient condition for a general pair of positive LTI systems to have a CDLF in Section 5. Two simple applications of this result are also given.

\section{MATHEMATICAL PRELIMINARIES}

Throughout, $\mathbb{R}$ denotes the field of real numbers, $\mathbb{R}^{n}$ stands for the vector space of all $n$-tuples of real numbers and $\mathbb{R}^{m \times n}$ is the space of $m \times n$ matrices with real entries. For $x$ in $\mathbb{R}^{n}, x_{i}$ denotes the $i^{t h}$ component of $x$, and the notation $x \succ 0(x \succeq 0)$ means that $x_{i}>0\left(x_{i} \geq 0\right)$ for $1 \leq i \leq n$. Similarly, for a matrix $A$ in $\mathbb{R}^{n \times n}, a_{i j}$ denotes the element in the $(i, j)$ position of $A$, and $A \succ 0(A \succeq 0)$ means that $a_{i j}>0\left(a_{i j} \geq 0\right)$ for $1 \leq i, j \leq n$. $A \succ B$ ( $A \succeq B)$ means that $A-\bar{B} \succ 0(A-\bar{B} \succeq 0)$. We write $A^{T}$ for the transpose of $A$. For $P$ in $\mathbb{R}^{n \times n}$ the notation $P>0$ means that the matrix $P$ is positive definite.

The spectral radius of a matrix $A$ is the maximum modulus of the eigenvalues of $A$ and is denoted by $\rho(A)$. Also we shall denote the maximal real part of any eigenvalue of $A$ by $\mu(A)$. If $\mu(A)<0, A$ is said to be Hurwitz. Finally for two matrices, $A, B \in \mathbb{R}^{n \times m}$, we denote the entrywise or Hadamard product [21] of $A$ and $B$ by $A \circ B$.

\section{Positive LTI systems and Metzler matrices:}

The LTI system

$$
\Sigma_{A}: \dot{x}(t)=A x(t), \quad x(0)=x_{0}
$$

is said to be positive if $x_{0} \succeq 0$ implies that $x(t) \succeq 0$ for all $t \geq 0$. See [3] for a description of the basic theory and several applications of positive linear systems.

It is well-known that the system $\Sigma_{A}$ is positive if and only if the off-diagonal entries of the matrix $A$ are non-negative. Matrices of this form are known as Metzler matrices. If $A$ is Metzler we can write $A=N-\alpha I$ for some non-negative $N$ and a scalar $\alpha \geq 0$, and the Metzler matrix $N-\alpha I$ will be Hurwitz if and only if $\alpha>\rho(N)$. Metzler Hurwitz matrices are closely related to the class of so-called M-matrices [2], [21]. In fact, a matrix $A$ in $\mathbb{R}^{n \times n}$ is an M-matrix if and only if $-A$ is Metzler and Hurwitz.

We now record some fundamental properties of irreducible Metzler matrices corresponding to those established for non-negative matrices in the classical Perron-Frobenius Theorem [2]. For the definition of an irreducible matrix, see [22].

Theorem 2.1: Let $A=N-\alpha I \in \mathbb{R}^{n \times n}$ be Metzler and irreducible. Then

(i) $\mu(A)=\rho(N)-\alpha$ is an eigenvalue of $A$ of algebraic (and geometric) multiplicity one;

(ii) There is an eigenvector $x \succ 0$ with $A x=\mu(A) x$.

The next result concerning positive combinations of Metzler Hurwitz matrices was pointed out in [21].

Lemma 2.1: Let $A_{1}, A_{2}$ be Metzler and Hurwitz. Then $A_{1}+\gamma A_{2}$ is Hurwitz for all $\gamma>0$ if and only if $A_{1}+\gamma A_{2}$ is non-singular for all $\gamma>0$. (Equivalently $A_{1} A_{2}^{-1}$ has no negative real eigenvalues.)

\section{A CONJECTURE ON CQLF EXISTENCE FOR POSITIVE LTI SYSTEMS}

In [10], it was conjectured that given two stable positive LTI systems $\Sigma_{A_{1}}, \Sigma_{A_{2}}$, the matrix product $A_{1} A_{2}^{-1}$ having no negative eigenvalues was a necessary and sufficient condition for:

(i) the existence of a CQLF for the LTI systems $\Sigma_{A_{1}} \Sigma_{A_{2}}$;

(ii) global exponential stability, under arbitrary switching, of the associated switched linear system (1).

\section{A. Second order systems}

We now show that the above conjecture is true for second order systems. To begin with we recall the result of [8] which described necessary and sufficient conditions for the existence of a CQLF for a pair of general second order LTI systems.

Theorem 3.1: Let $A_{1}, A_{2} \in \mathbb{R}^{2 \times 2}$ be Hurwitz. Then a necessary and sufficient condition for $\Sigma_{A_{1}}, \Sigma_{A_{2}}$ to have a CQLF is that the matrix products $A_{1} A_{2}$ and $A_{1} A_{2}^{-1}$ have no negative eigenvalues.

We next show that it is only necessary to check one of the products in the above theorem if the individual systems 
$\Sigma_{A_{1}}, \Sigma_{A_{2}}$ are positive systems.

Lemma 3.1: Let $A_{1}, A_{2} \in \mathbb{R}^{2 \times 2}$ be Hurwitz and Metzler. Then the product $A_{1} A_{2}$ has no negative eigenvalue.

Proof: First of all, as $A_{1}, A_{2}$ are both Hurwitz, the determinant of $A_{1} A_{2}$ must be positive. Secondly, a straightforward calculation shows that all of the diagonal entries of $A_{1} A_{2}$ must be positive. Hence the trace of $A_{1} A_{2}$ is also positive. It now follows easily that the product $A_{1} A_{2}$ cannot have any negative eigenvalues as claimed.

A straightforward combination of Theorem 3.1 and Lemma 3.1 yields the following result.

Theorem 3.2: Let $\Sigma_{A_{1}}, \Sigma_{A_{2}}$ be stable positive LTI systems with $A_{1}, A_{2} \in \mathbb{R}^{2 \times 2}$. Then $A_{1} A_{2}^{-1}$ having no negative eigenvalues is a necessary and sufficient condition for $\Sigma_{A_{1}}$, $\Sigma_{A_{2}}$ to have a CQLF.

Comment: The above result establishes that if two second order positive LTI systems $\Sigma_{A_{1}}, \Sigma_{A_{2}}$ have no CQLF, there is some $\gamma>0$ for which $A_{1}+\gamma A_{2}$ is non-Hurwitz. It now follows from results presented in [23] that there exists some switching sequence for which the associated switching system

$$
\Sigma_{S}: \dot{x}=A(t) x \quad A(t) \in\left\{A_{1}, A_{2}\right\}
$$

is not exponentially stable. Thus for this class of switching system CQLF existence is equivalent to exponential stability under arbitrary switching.

Note that Theorem 3.2 implies that $A_{1} A_{2}^{-1}$ having no negative real eigenvalues is also a sufficient condition for $\Sigma_{A_{1}}, \Sigma_{A_{2}}$ to have a common quadratic copositive Lyapunov function also. On the other hand, if $A_{1} A_{2}^{-1}$ has a negative eigenvalue, then there exist $\gamma>0, \lambda \geq 0$ and a vector $x \succ 0$ such that $\left(A_{1}+\gamma A_{2}\right) x=\lambda x$. It follows from this that there cannot be a common quadratic copositive Lyapunov function for $\Sigma_{A_{1}}, \Sigma_{A_{2}}$. Thus, we have the following result.

Theorem 3.3: Let $A_{1}, A_{2}$ be Metzler, Hurwitz matrices in $\mathbb{R}^{2 \times 2}$. Then there exists a quadratic common copositive Lyapunov function for the systems $\Sigma_{A_{1}}, \Sigma_{A_{2}}$ if and only if $A_{1} A_{2}^{-1}$ has no negative real eigenvalues.

\section{B. Higher order systems}

The above conjecture arose from considering the situation where two stable positive LTI systems $\Sigma_{A_{1}}, \Sigma_{A_{2}}$ have no CQLF but there is a solution $P=P^{T} \geq 0$ to

$$
A_{i}^{T} P+P A_{i}=Q_{i} \leq 0 \quad i \in\{1,2\} .
$$

Numerical testing indicated that the matrices $Q_{i}$ occurring above were of rank $n-1$. Based on this, we believed that it would be possible to prove the conjecture following similar arguments to those used successfully to derive conditions for second order systems and systems in companion form in [14], [24]. Unfortunately this is not the case and we now present a counterexample to the conjecture.

Example 3.1: Consider the two Hurwitz matrices $A_{1}, A_{2} \in$ $\mathbb{R}^{3 \times 3}$

$$
\begin{aligned}
A_{1} & =\left(\begin{array}{ccc}
-0.5302 & 0.0012 & 0.0873 \\
0.2185 & -0.7494 & 0.5411 \\
0.7370 & 0.1543 & -0.3606
\end{array}\right) \\
A_{2} & =\left(\begin{array}{ccc}
-0.5136 & 0.4419 & 0.3689 \\
0.1840 & -0.3951 & 0.0080 \\
0.3163 & 0.6099 & -1.0056
\end{array}\right) .
\end{aligned}
$$

The eigenvalues of the product $A_{1} A_{2}^{-1}$ are given by $\{-0.8217+0.0835 i,-0.8217-0.0835 i, 2.2234\}$. However the systems $\Sigma_{A_{1}}, \Sigma_{A_{2}}$ have no CQLF as can be verified by showing that there exist positive semi-definite matrices $P_{1}, P_{2}$ satisfying $A_{1}^{T} P_{1}+P_{1} A_{1}+A_{2}^{T} P_{2}+P_{2} A_{2}>0$ [25] using the standard MATLAB LMI toolbox. Thus the condition that $A_{1} A_{2}^{-1}$ has no negative eigenvalues is not sufficient for CQLF existence.

Furthermore, in a recent paper [26], it has been shown that it is possible for $A_{1} A_{2}^{-1}$ to have no negative real eigenvalues, and for the associated positive switched linear system to fail to be exponentially stable under arbitrary switching. While the conjecture of [10] is not true for general systems, two points are worthy of note. Firstly, counterexamples to the conjecture appear to be quite rare and hence there is a possibility that related, simple necessary and sufficient conditions may be derived for CQLF existence for positive LTI systems. This is currently the subject of ongoing research by the authors. Secondly, we shall see in the next section that the rationale that led to the conjecture can be successfully applied to derive algebraic necessary and sufficient conditions for general pairs of positive LTI systems to have CDLFs.

\section{Sufficient CONDITIONS FOR CDLF EXISTENCE}

In this section, we turn our attention to obtaining stability criteria for positive switched linear systems based on the existence of CDLFs. In particular, in Theorem 4.1 below, we present a number of simple sufficient conditions for a pair of stable positive LTI systems to have a CDLF. For a proof of this theorem, consult [28].

Theorem 4.1: Let $A_{1}, A_{2}$ be Metzler, Hurwitz matrices in $\mathbb{R}^{n \times n}$. Then the following conditions are all sufficient for the existence of a CDLF for the LTI systems $\Sigma_{A_{1}}, \Sigma_{A_{2}}$.

(i) $-A_{1} A_{2}^{-1}$ and $-A_{2}^{-1} A_{1}$ are both Metzler and Hurwitz;

(ii) $A_{1} A_{2}^{-1}$ and $A_{2}^{-1} A_{1}$ are both non-negative matrices;

(iii) there is some finite collection of columns $c_{i_{1}}, c_{i_{2}}, \ldots, c_{i_{m}}$ taken from $A_{1} A_{2}^{-1}$, and some finite collection of rows $r_{j_{1}}^{T}, r_{j_{2}}^{T}, \ldots, r_{j_{l}}^{T}$ taken from 
$A_{2}^{-1} A_{1}$ such that

$$
\begin{aligned}
c_{i_{1}}+c_{i_{2}}+\cdots+c_{i_{m}} & \succ 0, \\
r_{j_{1}}+r_{j_{2}}+\cdots+r_{j_{l}} & \succ 0 ;
\end{aligned}
$$

(iv) $A_{1}^{T} A_{2}+A_{2}^{T} A_{1}>0$ and $A_{1} A_{2}^{T}+A_{2} A_{1}^{T}>0$.

Note that conditions (i) and (ii) of Theorem 4.1 were previously reported in [10] and that Ooba and Funahashi have derived a stronger version of condition (iv) for CDLF existence for pairs of discrete-time positive LTI systems in [27].

\section{NECESSARY AND SUFFICIENT CONDITIONS FOR CDLF EXISTENCE}

In this section, we continue to focus on the CDLF existence question and we shall see how the ideas that led to the conjecture discussed in section III can be successfully applied to derive a compact necessary and sufficient condition for the existence of a CDLF for a generic pair of positive LTI systems. In this regard, the result of Corollary 5.1 is particularly noteworthy as it establishes that for an irreducible Metzler, Hurwitz $A$ in $\mathbb{R}^{n \times n}$, if $D \geq 0$ is diagonal and $A^{T} D+D A=Q \leq 0$, then the rank of $Q$ must be $n-1$.

To begin with we present a number of technical preliminary results that are required in the proof of the main result of this section, which is given in Theorem 5.2 below.

\section{A. Preliminary results on Metzler matrices and diagonal Lyapunov functions}

For detailed proofs of the lemmas in this subsection, consult [28]. The following simple result is easily verified by direct computation.

Lemma 5.1: Let $A \in \mathbb{R}^{n \times n}$ be a Metzler matrix. Then for any non-zero diagonal matrix $D \geq 0$ in $\mathbb{R}^{n \times n}, A^{T} D+D A$ is also Metzler.

The next result is concerned with diagonal matrices $D \geq 0$ such that $A^{T} D+D A \leq 0$, and establishes that any such matrix must in fact be positive definite if $A$ is irreducible.

Lemma 5.2: Let $A$ in $\mathbb{R}^{n \times n}$ be Metlzer, Hurwitz and irreducible. Suppose that $A^{T} D+D A \leq 0$ for some diagonal $D \geq 0$ in $\mathbb{R}^{n \times n}$. Then $D>0$.

The next result establishes that if $A \in \mathbb{R}^{n \times n}$ is Metzler, Hurwitz and irreducible, and $D \geq 0$ a diagonal matrix such that $A^{T} D+D A=Q \leq 0$, then $Q$ must be irreducible also.

Lemma 5.3: Let $A \in \mathbb{R}^{n \times n}$ be Metzler, Hurwitz and irreducible. Suppose that for some non-zero diagonal $D \geq 0$ in $\mathbb{R}^{n \times n}, A^{T} D+D A=Q \leq 0$. Then $Q$ is also irreducible.

As an immediate consequence of the previous results, we have the following surprising corollary.
Corollary 5.1: Let $A \in \mathbb{R}^{n \times n}$ be Metzler, Hurwitz and irreducible. Suppose that $D \in \mathbb{R}^{n \times n}$ is diagonal, $D \geq 0$, and that $A^{T} D+D A=Q \leq 0$. Then $\operatorname{rank}(Q)=n-1$, and there is some vector $v \succ 0$ such that $Q v=0$.

Proof: It follows from Lemma 5.1 and Lemma 5.3 that $Q$ is an irreducible Metzler matrix. Furthermore, as $Q \leq$ $0, \mu(Q)=0$. The result now follows immediately from Theorem 2.1.

\section{Necessary conditions:}

Finally for this subsection, we present a simple necessary condition for a pair of LTI systems to have a CDLF, and a corresponding necessary condition for a single stable LTI system to have a diagonal Lyapunov function. Note how the condition of the next lemma is related to the necessary conditions previously established for the general CQLF problem (see for instance [14]).

Lemma 5.4: Let $A_{1}, A_{2}$ be Hurwitz matrices in $\mathbb{R}^{n \times n}$ such that $\Sigma_{A_{1}}, \Sigma_{A_{2}}$ have a CDLF. Then for all non-singular diagonal matrices $D$ in $\mathbb{R}^{n \times n}, A_{1}+D A_{2} D$ and $A_{1}^{-1}+$ $D A_{2} D$ are Hurwitz and hence non-singular.

The fact that $\Sigma_{A}$ has a diagonal Lyapunov function if and only if $\Sigma_{A}, \Sigma_{A^{-1}}$ have a CDLF leads to the following necessary conditions for a single stable LTI system to have a diagonal Lyapunov function.

Corollary 5.2: Let $\Sigma_{A}$ be a stable LTI system with $A \in$ $\mathbb{R}^{n \times n}$. Then a necessary condition for $\Sigma_{A}$ to have a diagonal Lyapunov function is that $A+D A^{-1} D$ and $A+D A D$ are Hurwitz for all non-singular diagonal matrices $D \in$ $\mathbb{R}^{n \times n}$.

\section{B. The main result}

We now consider a pair of stable positive LTI systems $\Sigma_{A_{1}}, \Sigma_{A_{2}}$, where $A_{1}, A_{2}$ in $\mathbb{R}^{n \times n}$ are Hurwitz, Metzler and irreducible. We shall derive below a necessary and sufficient condition for $\Sigma_{A_{1}}, \Sigma_{A_{2}}$ to have a CDLF that is related to the matrix pencil conditions obtained for the general CQLF existence problem in [14], [24].

The following theorem considers the situation of two systems $\Sigma_{A_{1}}, \Sigma_{A_{2}}$ for which there is no CDLF but for which there is a non-zero diagonal $D \geq 0$ satisfying

$$
\begin{aligned}
& A_{1}^{T} D+D A_{1} \leq 0 \\
& A_{2}^{T} D+D A_{2} \leq 0 .
\end{aligned}
$$

We shall see that in this situation, the necessary conditions of Lemma 5.4 are violated.

Theorem 5.1: Let $A_{1}, A_{2}$ in $\mathbb{R}^{n \times n}$ be Hurwitz, Metzler and irreducible. Assume that there is no CDLF for the associated LTI systems, $\Sigma_{A_{1}}, \Sigma_{A_{2}}$. Furthermore, suppose that there is some non-zero diagonal $D \geq 0$ satisfying (2). Then there 
exists a diagonal matrix $D_{0}>0$ such that $A_{1}+D_{0} A_{2} D_{0}$ is singular.

Proof: First of all, it follows from Corollary 5.1 that $Q_{1}=$ $A_{1}^{T} D+D A_{1}$, and $Q_{2}=A_{2}^{T} D+D A_{2}$ must both have rank $n-1$, and that we can choose vectors $x_{1} \succ 0, x_{2} \succ 0$ such that $Q_{1} x_{1}=0, Q_{2} x_{2}=0$.

The next step in the proof is to show that there can be no diagonal matrix $D^{\prime}$ with

$$
\begin{aligned}
& x_{1}^{T} D^{\prime} A_{1} x_{1}<0 \\
& x_{2}^{T} D^{\prime} A_{2} x_{2}<0 .
\end{aligned}
$$

For details consult [28].

As there is no diagonal solution to (3), (4) it can be shown that there is some constant $k>0$ such that

$$
x_{1}^{T} D^{\prime} A_{1} x_{1}=-k x_{2}^{T} D^{\prime} A_{2} x_{2}
$$

for all diagonal $D^{\prime}$ in $\mathbb{R}^{n \times n}$. In fact, we can take $k=1$ as we may replace $x_{2}$ with $x_{2} / \sqrt{k}$ if necessary.

On expanding out equation (5) (with $k=1$ ) and equating coefficients, it follows that

$$
x_{1} \circ A_{1} x_{1}=-x_{2} \circ A_{2} x_{2} .
$$

Now as $x_{1} \succ 0, x_{2} \succ 0$, there is some diagonal matrix $D_{0}>0$ such that $x_{2}=D_{0} x_{1}$. But then, it follows from (6) that $A_{2} x_{2}=-D_{0}^{-1} A_{1} x_{1}$ and hence that $\left(D_{0}^{-1} A_{1}+\right.$ $\left.A_{2} D_{0}\right) x_{1}=0$. This means that $\operatorname{det}\left(A_{1}+D_{0} A_{2} D_{0}\right)=0$ which completes the proof.

We can now apply Lemma 5.4 and Theorem 5.1 to derive the main result of this section.

Theorem 5.2: Let $A_{1}, A_{2}$ in $\mathbb{R}^{n \times n}$ be Hurwitz, Metzler and irreducible. Then a necessary and sufficient condition for the positive LTI systems, $\Sigma_{A_{1}}, \Sigma_{A_{2}}$, to have a CDLF is that $A_{1}+D A_{2} D$ is non-singular for all diagonal $D>0$.

Proof: The necessity was proven in Lemma 5.4. Now suppose that there is no CDLF for $\Sigma_{A_{1}}, \Sigma_{A_{2}}$. Then,

(i) For $\alpha>0$ sufficiently large, $\Sigma_{A_{1}-\alpha I}, \Sigma_{A_{2}}$ will have a CDLF.

(ii) If we define

$$
\alpha_{0}=\inf \left\{\alpha: \Sigma_{A_{1}-\alpha I}, \Sigma_{A_{2}} \text { have a CDLF }\right\},
$$

then $\Sigma_{A_{1}-\alpha_{0} I}, \Sigma_{A_{2}}$ satisfy the conditions of Theorem 5.1 .

(iii) It follows that there is some diagonal $D>0$ such that $A_{1}-\alpha_{0} I+D A_{2} D$ is singular.

From item (iii), it follows that $A_{1}+D A_{2} D$ is not Hurwitz. However both $A_{1}$ and $D A_{2} D$ are Hurwitz Metzler matrices, and it therefore follows from Lemma 2.1 that there is some positive $\gamma>0$ such that $A_{1}+\gamma D A_{2} D$ is singular. Hence, defining $\bar{D}=\sqrt{\gamma} D$, we have that $A_{1}+\bar{D} A_{2} \bar{D}$ is singular. This completes the proof of the theorem.

\section{Comment:}

The condition of Theorem 5.2 can be thought of as a multivariable matrix pencil condition for CDLF existence and, as such it relates to the conditions for CQLF existence already derived in [14], [8]. Furthermore, to the best of the authors' knowledge Theorem 5.2 is the first result of its kind, giving necessary and sufficient conditions for CDLF existence for systems of arbitrary dimension. While the condition is extremely difficult to check, it is hoped that the insights provided by the result will lead to the derivation of simpler conditions for CDLF existence that may be used in the design of positive switched linear systems. This is currently the subject of ongoing research and future advances will be published as they are made.

\section{Two simple applications}

We now present two simple applications of Theorem 5.2. First of all, note the following easily verifiable facts.

(i) It follows from Corollary 5.2 that $D A_{1} D+A_{1}$ is Hurwitz and Metzler for all diagonal $D>0$.

(ii) If $B$ is any Metzler matrix with $A_{1} \succeq B$, then $B$ is also Hurwitz [21].

(iii) If $A_{1} \succeq B$, then for any diagonal $D>0, D A_{1} D+$ $A_{1} \succeq \bar{D} A_{1} D+B$.

Thus if $A_{1} \succeq A_{2}$, it follows from item (iii) that for all positive diagonal $D, D A_{1} D+A_{1} \succeq D A_{1} D+A_{2}$. Hence from (i) and (ii) it follows that $D A_{1} D+A_{2}$ is Hurwitz for all diagonal $D>0$. Thus applying Theorem 5.2 we have the following known result [10], [29].

Theorem 5.3: Let $A_{1}, A_{2} \in \mathbb{R}^{n \times n}$ be Metzler, Hurwitz and irreducible, and suppose $A_{1} \succeq A_{2}$. Then the positive LTI systems, $\Sigma_{A_{1}}, \Sigma_{A_{2}}$, have a CDLF.

It is in fact possible to slightly strengthen Theorem 5.3 by noting that, for a fixed diagonal $D_{1}>0$, as $D$ ranges over all positive diagonal matrices, so too does $D D_{1}=D_{1} D$. So if we know that $D D_{1} A_{1} D_{1} D+A_{2}$ is non-singular for all positive diagonal $D$, then $D A_{1} D+A_{2}$ is also non-singular for all positive diagonal $D$. This gives us the following result.

Corollary 5.3: Let $A_{1}, A_{2} \in \mathbb{R}^{n \times n}$ be Metzler, Hurwitz and irreducible. Suppose that for some diagonal $D_{1}>0$, $D_{1} A_{1} D_{1} \succeq A_{2}$. Then the positive LTI systems, $\Sigma_{A_{1}}, \Sigma_{A_{2}}$, have a CDLF.

\section{CONCLusions}

In this paper we presented a number of preliminary results on the stability of positive switched linear systems. In particular, we have shown that a recently made conjecture concerning CQLF existence for pairs of positive LTI systems is true for second order systems, and that for the related 
class of positive switched linear systems, CQLF existence is equivalent to exponential stability under arbitrary switching. We have also presented a counterexample showing that the conjecture is in general untrue for higher dimensional systems. However, the underlying ideas that led to the conjecture have been used to derive a new algebraic condition that is necessary and sufficient for CDLF existence for pairs of positive LTI systems of arbitrary dimension. A number of simple stability criteria for positive switched linear systems based on the existence of CDLFs have also been presented.

Acknowledgements: This work was partially supported by Science Foundation Ireland grant 00/PI.1/C067, Science Foundation Ireland Basic Research Grant 04/BR/m0061 and Enterprise Ireland grant SC/2000/084/Y. Neither Science Foundation Ireland nor Enterprise Ireland is responsible for any use of data appearing in this publication.

\section{REFERENCES}

[1] D. Luenberger, Introduction to Dynamic Systems: Theory, Models, and Applications. Wiley, 1979.

[2] A. Berman and R. Plemmons, Non-negative matrices in the mathematical sciences. SIAM Classics in Applied Mathematics, 1994.

[3] L. Farina and S. Rinaldi, Positive linear systems. Wiley Interscience Series, 2000.

[4] T. Haveliwala and S. Kamvar, "The second eigenvalue of the Google matrix," tech. rep., Stanford University, March 2003.

[5] R. Shorten, D. Leith, J. Foy, and R. Kilduff, "'towards an analysis and design framework for congestion control in communication networks," in Proceedings of the 12th Yale workshop on adaptive and learning systems, 2003.

[6] A. Jadbabaie, J. Lin, and A. S. Morse, "Co-ordination of groups of mobile autonomous agents using nearest neighbour rules," IEEE Transactions on Automatic Control, vol. 48, no. 6, pp. 988-1001, 2003.

[7] W. P. Dayawansa and C. F. Martin, "A converse Lyapunov theorem for a class of dynamical systems which undergo switching," IEEE Transactions on Automatic Control, vol. 44, no. 4, pp. 751-760, 1999.

[8] R. N. Shorten and K. Narendra, "Necessary and sufficient conditions for the existence of a common quadratic Lyapunov function for a finite number of stable second order linear time-invariant systems," International Journal of Adaptive Control and Signal Processing, vol. 16, p. 709.

[9] R. N. Shorten and K. S. Narendra, "On common quadratic lyapunov functions for pairs of stable lti systems whose system matrices are in companion form," IEEE Transactions on Automatic Control, vol. 48, no. 4, pp. 618-621, 2003.

[10] O. Mason and R. Shorten, "A conjecture on the existence of common quadratic Lyapunov functions for positive linear systems," in Proceedings of American Control Conference, 2003.

[11] V. Blondel, E. Sontag, M. Vidyasagar, and J. C. Willems, Open Problems in Mathematical Systems and Control Theory. Springer, 1998.

[12] N. Oleng and K. Narendra, "On the existence of diagonal solutions to the Lyapunov equation for a third order system," in Proceedings of American Control Conference, 2003.

[13] J. Kraaijevanger, "A characterization of Lyapunov diagonal stability using Hadamard products," Linear Algebra and its Applications, vol. 151, pp. 245-254, 1991.

[14] R. N. Shorten, K. S. Narendra, and O. Mason, "On common quadratic Lyapunov functions," IEEE Transactions on Automatic Control, vol. 48 , no. 1 , pp. $110-113,2003$.

[15] G. P. Barker, A. Berman, and R. J. Plemmons, "Positive Diagonal Solutions to the Lyapunov Equations," Linear and Multilinear Algebra, vol. 5, no. 3, pp. 249-256, 1978.

[16] G. W. Cross, "Three types of matrix stability," Linear Algebra and its Applications, vol. 20, pp. 253-263, 1978.
[17] R. Redheffer, "Volterra multipliers (i) and (ii)," SIAM J. Alg. Disc. Meth., vol. 6, no. 4, pp. 592-623, $1985 \mathrm{~s}$.

[18] D. Siljak, Decentralized control of complex systems. Academic Press, San Diego, 1991.

[19] M. Vidyasagar, "New directions of research in nonlinear system theory," Proceedings of the IEEE, vol. 74, no. 8, pp. 1060 - 1091, 1986.

[20] E. Kaszkurewicz and A. Bhaya, Matrix diagonal stability in systems and computation. Birkhauser, 1999.

[21] R. Horn and C. Johnson, Topics in matrix analysis. Cambridge University Press, 1991.

[22] R. Horn and C. Johnson, Matrix analysis. Cambridge University Press, 1985.

[23] R. Shorten, F. Ó Cairbre, and P. Curran, "On the dynamic instability of a class of switching systems," in Proceedings of IFAC conference on Artificial Intelligence in Real Time Control, 2000.

[24] R. N. Shorten, O. Mason, F. O. Cairbre, and P. Curran, "A unifying framework for the SISO Circle Criterion and other quadratic stability criteria," International Journal of Control, vol. 77, no. 1, pp. 1-8, 2004.

[25] S. Boyd, L. El Ghaoui, E. Feron, and V. Balakrishnan, Linear Matrix Inequalities in System and Control Theory. Philadelphia: SIAM, 1994.

[26] L. Gurvits, R. Shorten, and O. Mason, "Preliminary results on the stability of switched positive linear systems," Submitted to Mathematical Theory of Networks and Systems, 2004.

[27] T. Ooba and Y. Funahashi, "On the simultaneous diagonal stability of linear discrete-time systems," Systems and Control Letters, vol. 36, pp. 175-180, 1999.

[28] O. Mason, Switched systems, convex cones and common Lyapunov functions. PhD thesis, Department of Electronic Engineering, National University of Ireland, Maynooth, 2004.

[29] Y. Mori, T. Mori, and Y. Kuroe, "On a class of linear constant systems which have a common quadratic Lyapunov function," in Proceedings of the IEEE Conference on Decision and Control, 1998. 